\title{
ARBORIZACIÓN URBANA: UNA SITUACIÓN EN CAMPOS DOS GOYTACAZES
}

\author{
Verônica de Morais \\ Especialista en Plantas Ornamentales y Paisajismo/UFLA/MG \\ ${ }^{1}$ Professor del Instituto Superior de Tecnología en Ciencias Agrarias - FAETEC \\ veronicade@ig.com.br
}

Cláudio Luiz Melo de Souza

Dsc. en Produción Vegetal/UENF/RJ

${ }^{1,2}$ Professor del Instituto Superior de la Educación Nossa Senhora Auxiliadora - ISECENSA

ist.campos@faetec.rj.gov.br

Paulo Roberto Corrêa Landgraf

Dsc. en Fitotecnia/UFLA/MG

Laboratório de sementes/UNIFENAS/MG

plandgraf@gmail.com

\section{RESUMEN}

La arborización urbana forman parte del ecosistema de las ciudades, promoviendo beneficios que merecen la atención del poder público. La arborización sin planeamiento genera serios problemas de incompatibilidad con los paseos y equipamientos públicos, muros, redes hidráulicas, eléctricas y de telecomunicaciones. Este estúdio tiene como objetivo hacer el levantamiento e identificar las condiciones de la arborización en las inmediaciones de la Plaza Barão do Rio Branco (Plaza del Liceo de Humanidades) en el Barrio Jardín Maria de Queiroz, Campos dos Goytacazes, Rio de Janeiro, Brasil. Los parámetros para la recopilación de datos fueron, la identificación de las especies, origen, situación de la raíz, área libre del entorno de la raíz, altura de la primera bifurcación, tipo de poda y situación de conflicto de la copa con la red de cables eléctricos del área. Fueron 3registrados 151 indivíduos pertenecientes a 25 especies, distribuidos en 23 géneros y 15 familias botánicas. Se constató una relación de 1 especie nativa para 1,5 especies exóticas. Los árboles que presentaron raíces expuestas que dañaban las aceras totalizaron $59 \%$. La disponibilidad del área del entorno de las plantas no fue respetada (no existe) en $84,11 \%$ de los casos, el 8,61\% de los árboles de esa área era $\geq 1,00 \mathrm{~m}^{2}$, y en el 7,28\% de los casos era $<1,00 \mathrm{~m}^{2}$. En el 33\% de los árboles a la altura de la primera bifurcación estaba por encima de 1,80 $\mathrm{m}$ del suelo; $50,33 \%$ presentaban algún tipo de poda y de esas fueron consideradas mutilaciones el 15,23\%. En el $10,60 \%$ de los casos, los árboles impedian el paso de los peatones en las aceras y el $31,79 \%$ estaban en conflicto con los cables eléctricos del área.

Palabras-clave: Levantamiento, Arborización Urbana.

\begin{abstract}
Urban forestry is integrated the ecosystems of the cities, promoting important benefices which deserve attention of the public administration. When the forestry is not planning, it can cause serious problems such as destruction of sidewalks, public equipamenties, hydraulic nets, electrical cabs or web cabs. The objective of this study was to quantify and determine the botanical species used for urban forestry in mediations of Barão do Rio Branco Plaza of the district of Maria de Queiroz garden, Campos dos Goytacazes, Rio de Janeiro, Brazil. The datas collected about the trees were: the botanical identification, the geographic origin, the roots conditions, the perimeter of soil for roots, the chest height, the conflicts between tops tree and electrical cabs, as well as, the conflicts between roots and sidewalks. This study recorded in the locality, 151 trees of 25 species belonging to
seer.perspectivasonline.com.br

Volume 1. número 1, 2011
\end{abstract}


23 generals and 15 botanical families. About the origin center, $57.72 \%$ of trees were native from Brazil and $42.28 \%$ were exotic. The trees with roots destructing the sidewalks, it occurred in $36 \%$ of cases. The perimeter of soil for roots were not observed to $84.11 \%$ of trees. About the chest height, $33 \%$ of trees were bellow of 1.80 $\mathrm{m}$. The trees with tops courted, it occurred in $33 \%$ of cases, and in $15.23 \%$ it were courted drastically. Around the $10.60 \%$ of trees were difficulting the pedestrian's transit and $31.79 \%$ of trees were improperly in contact with electrical cabs.

Key words: Inventory, Urban Forestry.

\section{INTRODUCCIÓN}

Una arborización correcta y armoniosa, al mismo tiempo en que refleja la cultura y el grado de civilización de una ciudad, constituye uno de los más sólidos elementos de su valorización, promoviendo mejorías en la calidad de vida y tornando el ambiente más agradable. Ella tiene influencia en el mantenimiento del micro clima, el equilibrio de la temperatura y la iluminación, la reducción de la contaminación atmosférica y sonora, el equilibrio hídrico y la amortización del impacto de la lluvia y la reducción de la erosión. (GUIZO y JASPER, 2005).

La arborización urbana se caracteriza por ser uno de los más importantes elementos que componen el ecosistema urbano y que, por los beneficios que produce, merece mayor atención en cualquier planeamiento urbano. La ausencia de una política sistemática y planeada con criterios paisajísticos genera serios problemas a los usuarios urbanos en lo referente a incompatibilidades con la movilidad, en especial, para las aceras y paseos públicos, muros, redes hidráulicas, eléctricas y de telecomunicaciones (CEMIG, 2001).

Otros importantes factores estan relacionados con las características botánicas indeseables, tales como la presencia de espinas, de principios alérgicos y tóxicos que necesitan de especial planeamiento. La ausencia de principios tóxicos es uno de los factores más importantes en la selección de especies para el entorno urbano. (BIONDI y ALTHAUS,2005).

Además, existen diferentes propiedades botánicas que pueden ser beneficiosas o tener inconvenientes y ser inapropiados para el lugar donde se coloquen, tales como, el tamaño de la especie, el tipo de flores y frutos, el crecimiento de las raíces y la caída de las hojas y tallos, además de la atracción de fauna indeseable como por ejemplo los murcielagos. (PRADO y PAIVA, 2001)

Este trabajo tiene como objetivo, establecer un diagnóstico específico de la arborización destinada a las calles circundantes a la Plaza Rio Branco, en el Municipio Fluminense de Campos dos Goytacazes, donde estan situadas muchas edificaciones históricas y públicas con un gran flujo de usuarios y residentes. El diagnóstico puede ser considerado como pionero y podrá ofrecer informaciones básicas para un futuro trabajo de arborización tanto para entidades públicas como para la población en general.

\section{MATERIAL Y MÉTODOS}

Localización del área: La ciudad de Campos dos Goytacazes está situada al Norte del Estado de Rio de Janeiro, apróximadamente a $15 \mathrm{~m}$ sobre el nivel del mar, tiene una población de 416,212 habitantes, área de $4.031 .910 \mathrm{~km}^{2}$ y una densidad de 107,64 habitantes $/ \mathrm{km}^{2}$. La temperatura media anual es de $23,2^{0} \mathrm{C}$, siendo Julio el mes más frio y Febrero el más caliente. La media anual de precipitación pluviométrica es de $1.087 \mathrm{~mm}$, con los meses más lluviosos entre Noviembre y Enero. El invierno es seco con periodo crítico entre Junio y Agosto (IBGE, 2009). El sitio fue escogido en función de los problemas observados en las calles de acceso a la Plaza

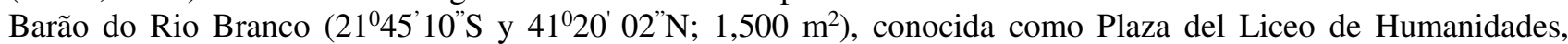
importante patrimônio histórico-arquitectónico de la ciudad, destacándose el edificio de la Cámara Municipal que retrata el Partenón de Atenas y el Palacete del Liceo de Humanidades, donde vivió el Barão da Lagoa Dourada. Las secciones de las calles Barão da Lagoa Dourada, Baronesa da Lagoa Dourada, Baltazar Carneiro, Alberto Torres, Gil Góis, Salvador Corréa y Manhães Barreto. 
Muestreo: Este estudio fue realizado en el mes de Octubre del 2009, las calles en estudio son asfaltadas y fueron evaluados los árboles y arbustos presentes en las aceras de ambos lados. Los parámetros evaluados están de acuerdo con SILVA (2003), como sigue.

Identificación botánica: Los nombres comunes y científicos de las especies fueron identificados in loco y otros por la comparación de muestras vegetales con colecciones de los herbarios de lo Instituto Superior de Tecnologia em Ciências Agrárias. La clasificación de las familias botánicas siguió las directrices de Angiosperm Phylogeny Group (APG 11).

Origen y aspectos botánicos: Por medio de la bibliografía botánica especializada (LORENZI, 2008 a, b; LORENZI et al., 2003), fueron caracterizadas las especies por su porte, su utilización, su época de florecimiento, el color de las flores y su origen geográfico. Fueron consideradas nativas, las especies originarias del ecosistema del territorio brasileño y exóticas las de otro ecosistema.

Condiciones de las raíces: Fueron observadas las condiciones de (1) raíces cubiertas no problemáticas (sin daño a las aceras próximas) y (2) raíces expuestas problemáticas. Otras combinaciones no fueron observadas.

Régimen de zona: Se refiere al área de tierra disponible para el engrosamiento del tronco del árbol y la infiltración de agua, también llamada eco-anillo o collar. El área de suelo disponible para el entorno de las plantas fue clasificada asi: (1) no existe, (2) superior a $1 \mathrm{~m}^{2} \mathrm{o}(3)$ inferior a $1 \mathrm{~m}^{2}$.

Altura de la primera bifurcación: Con auxilio de la cinta métrica se evaluó la altura desde el suelo hasta el punto de inserción del primer tallo del tronco, determinándose las categorías: (1) superior e (2) inferior a $1 \mathrm{~m}^{2}$.

Tipo de poda: Los árboles fueron divididos en dos categorías: (1) no podados y (2) podados, siendo estas últimas sub divididas en podas convencionales con funciones de mantenimiento y limpieza, o mutilaciones que desequilibran el formato natural de la copa.

Conflictos de la copa o corona: Fueron analizadas las siguientes situaciones: (1) libres, cuando la copa no dificulta el paso de peatones y no tiene contacto con cables de las redes eléctricas o de comunicaciones ; (2) conflicto con el paso de peatones, y (3) conflicto con cables de la red eléctrica aérea.

Análisis estadístico: Los datos obtenidos fueron sometidos a análisis descriptivo por medio de la distribución de la frecuencia porcentual absoluta y relativa para las especies identificadas y los parámetros cualitativos investigados.

\section{RESULTADOS Y DISCUSIÓN}

En el estudio de los árboles fueron observados 151 individuos pertenecientes a 25 especies, distribuidos en 23 géneros y 15 familias botánicas (TABLA 1). La familia botánica que presentó mayor destaque, en riquezas de especies fue la Fabaceae, con nueve diferentes especies, y las familias con mayor frecuencia de individuos fueron, respectivamente, Fabaceae $(38,41 \%)$ y Chrysobalanaceae $(21,19 \%)$, juntas casi sumaron el $60 \%$ del total de individuos mostrados. La oiti, Licania tomentosa (Benth.) Fristch fue la única representante de la familia Chrysobalanaceae.

Las especies con mayor número de individuos fueron en orden decreciente: Caesalpinia pluviosa DC (25,17\%), L. tomentosa (Benth.) Fristsh (21,19\%), Murraya paniculata (L.) Jacq. (9,27\%), Bauhinia variegata L. $(6,62 \%)$ y Handroanthus chrysotrichus (Mart. E A. DC.) $(6,62 \%)$.

En un estudio realizado con los árboles del campo de la Universidad Federal de Brasilia, las leguminosas representaron el $32 \%$ de especies presentes y el $44 \%$ del número total de individuos. (KURIHARA et al, 2005). Teixeira (1999) también constató que de un total de 2,788 individuos pertenecientes a 112 especies y 14 eran de la familia Fabaceae (=Leguminosae).

La diversidad es importante para la cualidad ambiental, pero son escasos los estudios con propuestas de un método padrón para tales medidas en entornos urbanos (MILANO y DISPERATI, 1995). Grey y Deneke (1978) sugieren que el límite máximo de frecuencia este entre 10 y 15\% de individuos por especies, para reducir los problemas fitosanitarios.

En relación al origen geográfico de las especies, se comprobó que del total de las identificadas, 15 son exóticas y 10 nativas, siendo una relación de 1,5:1 (exótica:nativa). Por otra parte, 57,72\% del total de individuos mostrados, eran nativos (Tabla 1). Eso ocurrió debido al hecho de que muchas especies mostradas eran exóticas, pero en contrapartida hubo predominio de las especies nativas. L. tomentosa y $C$. pluviosa DC. $(=C$. 
peltophoroides). La diversidad de paisajismo urbano es considerada de gran importancia para la reducción de la monotonía del paisaje, proliferado de plagas y enfermedades, abrigo y alimentación de la fauna urbana, principalmente de pájaros (LANGOWSKI, 2001).

Tabla 1 - Levantamiento de árboles en las calles del Barrio Jardín Maria de Queiroz con acceso a la Plaza Barão do Rio Branco, Campos dos Goytacazes, RJ, 2009.

\begin{tabular}{|c|c|c|c|c|c|c|c|}
\hline \multirow{3}{*}{$\begin{array}{l}\text { Família } \\
\text { Especie/Nombres Vulgares } \\
\text { Brasileños }\end{array}$} & \multicolumn{5}{|c|}{ Principales Características Botánicas* } & \multirow{3}{*}{$\begin{array}{c}\mathrm{N}^{\mathrm{o}} \mathrm{de} \\
\text { casos } \\
(\mathrm{N}=151)\end{array}$} & \multirow{3}{*}{$(\%)$} \\
\hline & \multirow[b]{2}{*}{ Origen } & \multirow[b]{2}{*}{ Porte } & \multicolumn{2}{|c|}{ Floración } & \multirow[b]{2}{*}{ Hojas } & & \\
\hline & & & Época & Color & & & \\
\hline \multicolumn{8}{|l|}{ Anacardiaceae } \\
\hline Anacardium occidentale L. - cajueiro & $\mathrm{N}$ & $\mathrm{G}$ & $\begin{array}{l}\text { Jun- } \\
\text { nov }\end{array}$ & Rojo vino & $\mathrm{C}$ & 1 & 0,66 \\
\hline \multicolumn{8}{|l|}{ Apocynaceae } \\
\hline Nerium oleander L. - espirradeira & $\mathrm{E}$ & $\mathrm{P}$ & $\begin{array}{l}\text { Sept- } \\
\text { mar }\end{array}$ & Rosado & $\mathrm{P}$ & 1 & 0,66 \\
\hline \multicolumn{8}{|l|}{ Bignoniaceae } \\
\hline $\begin{array}{l}\text { Tecoma stans (L) Juss. Ex Kunth - } \\
\text { ipê-mirim }\end{array}$ & $\mathrm{E}$ & $\mathrm{P}$ & $\begin{array}{c}\text { Ene/M } \\
\text { ay }\end{array}$ & Amarillo & $\mathrm{P}$ & 6 & 3,97 \\
\hline $\begin{array}{l}\text { Handroanthus chrysotrichus (Mart. E } \\
\text { A. DC.) Mattos -ipê-amarelo }\end{array}$ & $\mathrm{N}$ & $\mathrm{G}$ & $\begin{array}{l}\text { Ago- } \\
\text { sept }\end{array}$ & Amarillo & $\mathrm{C}$ & 10 & 6,62 \\
\hline \multicolumn{8}{|l|}{ Combretaceae } \\
\hline $\begin{array}{l}\text { Terminalia catappa L. - chapéu-do- } \\
\text { sol }\end{array}$ & $\mathrm{E}$ & G & $\begin{array}{c}\text { Sept/oc } \\
\mathrm{t}\end{array}$ & Blanco & $\mathrm{C}$ & 1 & 0,66 \\
\hline \multicolumn{8}{|l|}{ Chrysobalanaceae } \\
\hline $\begin{array}{l}\text { Licania tomentosa (Benth.) Fritch. - } \\
\text { oiti }\end{array}$ & $\mathrm{N}$ & G & $\begin{array}{c}\text { Jun/Se } \\
\mathrm{pt}\end{array}$ & Blanco & $\mathrm{P}$ & 32 & 21,19 \\
\hline \multicolumn{8}{|l|}{$\begin{array}{l}\text { Fabaceae } \\
\text { (=Leguminosae:Caesalpinioideae) }\end{array}$} \\
\hline Bauhinia variegata L. - pata-de-vaca & $\mathrm{E}$ & M & Jul/Oct & Rosada & $\mathrm{C}$ & 10 & 6,62 \\
\hline Cassia fistula L. - chuva-de-ouro & $\mathrm{E}$ & G & $\begin{array}{l}\text { Nov- } \\
\text { Ene }\end{array}$ & Amarillo & $\mathrm{SC}$ & 1 & 0,66 \\
\hline Caesalpinia pluviosa DC. - sibiiruna & $\mathrm{N}$ & G & $\begin{array}{l}\text { Ago/N } \\
\text { ov }\end{array}$ & Amarillo & $\mathrm{SC}$ & 38 & 25,17 \\
\hline C. echinata Lam. - pau-brasil & $\mathrm{N}$ & G & $\begin{array}{l}\text { Sept- } \\
\text { oct }\end{array}$ & Amarillo & $\mathrm{SC}$ & 1 & 0,66 \\
\hline $\begin{array}{l}\text { C. pulcherrima (L) Sw. - flamboyant- } \\
\text { mirim }\end{array}$ & $\mathrm{N}$ & $\mathrm{P}$ & $\begin{array}{l}\mathrm{Sept} / \mathrm{Fe} \\
\mathrm{b}\end{array}$ & Rojo & $\mathrm{P}$ & 1 & 0,66 \\
\hline $\begin{array}{l}\text { Delonix regia (Bojer) Raf. - } \\
\text { flamboyant }\end{array}$ & $\mathrm{E}$ & M & $\begin{array}{l}\text { Oct/En } \\
\mathrm{e}\end{array}$ & Rojo & $\mathrm{SC}$ & 4 & 2,65 \\
\hline $\begin{array}{l}\text { Senna siamea Irwin y Barneby - cássia } \\
\text {-siamesa }\end{array}$ & $\mathrm{E}$ & G & $\begin{array}{l}\text { Ene- } \\
\text { Jun }\end{array}$ & Amarillo & $\mathrm{P}$ & 1 & 0,66 \\
\hline $\begin{array}{l}\text { Fabaceae (=Leguminosae: } \\
\text { Mimosoideae) }\end{array}$ & & & & & & & \\
\hline $\begin{array}{l}\text { Calliandra sp - esponja } \\
\text { Fabaceae (=Leguminosae: } \\
\text { (Papilonoideae) }\end{array}$ & $\mathrm{N}$ & $\mathrm{P}$ & Jul/Ago & Rosada & $\mathrm{P}$ & 1 & 0,66 \\
\hline $\begin{array}{l}\text { Clitoria fairchildiana R.A. Howard.- } \\
\text { sombreiro }\end{array}$ & $\mathrm{N}$ & G & $\begin{array}{c}\mathrm{Dic} / \mathrm{Ma} \\
\mathrm{y}\end{array}$ & Rojo vino & $\mathrm{C}$ & 1 & 0,66 \\
\hline
\end{tabular}



Ficus benjamina L.-ficus
Malpighiaceae

Malpighia glabra L. - acerola

Malvaceae

Hibiscus rosa-sinensis L.- hibisco

Pachira aquatica Aubl. - falso-cacau

$\begin{array}{lllllll}\text { E } & \text { G } & \text { Varios } & \text { Rojo } & \text { P } & 7 & 4,64 \\ \text { E } & \text { P } & \text { Varios } & \text { Rosado } & \text { P } & 2 & 1,32\end{array}$

Oxalidaceae

Averrhoa carambola L. - carambola

Polygonaceae

Triplaris brasiliana Cham. - pau-

formiga

$\begin{array}{lllllll}\text { E } & \text { P } & \text { Varios } & \text { Rosado } & \text { P } & 2 & 1,32 \\ \text { E } & \text { P } & \text { Varios } & \text { Rojo } & \text { P } & 1 & 0,66 \\ \text { N } & \text { G } & \text { Sept- } & \text { Blanco } & \text { P } & 1 & 0,66\end{array}$

Rutaceae

Murraya paniculata (L.) Jacq. -

falsa-murta

Salicaceae

Salix babylonica L. - chorão

$\begin{array}{ccclccc}\text { E } & \text { M } & - & \text { Rosado } & \text { P } & 1 & 0,66 \\ \text { N } & \text { G } & \begin{array}{c}\text { Ago- } \\ \text { oct }\end{array} & \begin{array}{l}\text { Rosa- } \\ \text { Anaranjad }\end{array} & \text { SC } & 6 & 3,97 \\ \text { E } & \text { P } & \begin{array}{c}\text { Oct/En } \\ \text { e }\end{array} & \text { Blanco } & \text { SC } & 14 & 9,27 \\ \text { E } & \text { M } & \begin{array}{c}\text { Todo el } \\ \text { Año }\end{array} & \text { Blanco } & \text { SC } & 5 & 3,31\end{array}$

Sapotaceae

Achras sapota L. - sapoti

E $G$

$\begin{array}{lll}\text { Blanco } & \mathrm{P} & 1\end{array}$

0,66

*Legenda: (N) Nativa, (E) Exótica, (P) Pequeño, (M) Medio, (G) Grande, (C) Caduca, (P) Perenne, (SC) Semi-Caduca. Las características botánicas fueron adaptadas de Paiva y Gavilanes, 2004; Lorenzi, 2008 a. b e c; Lorenzi et al. 2003.

Iguales datos fueron registrados en la Cachoeira do Sul (RS), donde el $58 \%$ de las especies de árboles utilizados en la arborización de áreas verdes fueron consideradas exóticas y $42 \%$ eran de ecosistémas locales. Sin embargo, entre la relación de la densidad de individuos, los números se mostraron diferentes, siendo los exóticos el 40\% y los nativos el 60\%. (LINDENMAIER y SANTOS, 2008). En Pernambuco, Campina Grande, se comprobó que el 58,9\% de las especies eran nativas y el 41,1\% eran exóticas, sin embargo el 67,2\% de individuos eran exóticos y 32,8\% eran nativos (DANTAS y SOUZA, 2004). Silva Junior y Correia (2001), observaron en el campo de la Universidad de Brasilia, que el 54\% de la especies eran nativas de la región central de Brasil y el otro $46 \%$ eran exóticas. Paiva et al. (2004) en la parcela central de la Universidad Federal de Lavras, identificaron 46 especies florales de un total de 182 ejemplares, pertenecientes a 24 familias. La especie Callistemon viminalis G. Don ex Loud, conocida como escova-de-garrafa, fue la que presentó mayor frecuencia (23 casos), seguida de ipê-roxo, Tabebuia impiteginosa (Mart.) Standl) (15 casos). Las nativas fueron valorizadas en la arborización en el $84 \%$ de los casos.

Las tendencias paisajísticas anteriores reflejan la utilización excesiva de especies exóticas, además, es más fácil encontrar especies de gran belleza por todo el mundo y no solamente en un espacio geográfico restringido. También hay un evidente desconocimiento, por parte de la población y organos guvernamentales, acerca de la utilización de especies brasileñas y son pocos los estudios sobre reservas forestales (LINDENMAIER y SANTOS, 2008).

En cuanto a exposición de las raíces, se observó que un 36\% estaban cubiertas y $64 \%$ estaban expuestas, de estas últimas, el 59\% ya presentaban algún tipo de daño en las aceras y apenas el 5\% no presentaban daños, aunque en un futuro pueden presentarlo (Figura 1).

El daño en las aceras, por raíces agresivas, no solo representa pérdidas materiales y estéticas, sino que reduce la movilidad de los transeuntes, dificultando el paso de niños, embarazadas, ancianos y personas con deficiencias. Esto al parecer, se trata de un problema generalizado en relación a la arborización urbana (SCHUCH, 2006; LINDERNMAIER y SANTOS, 2008; DEL CARO, 2009; MOURA y SANTOS, 2009).

PERSPECTIMASCOC
Lytraceae
Lagerstroemia indica L. - resedá

PERSPECTIVASCOC
Lytraceae
Lagerstroemia indica L. - resedá nov 


\section{Exposición de las raíces y daños en las aceras}
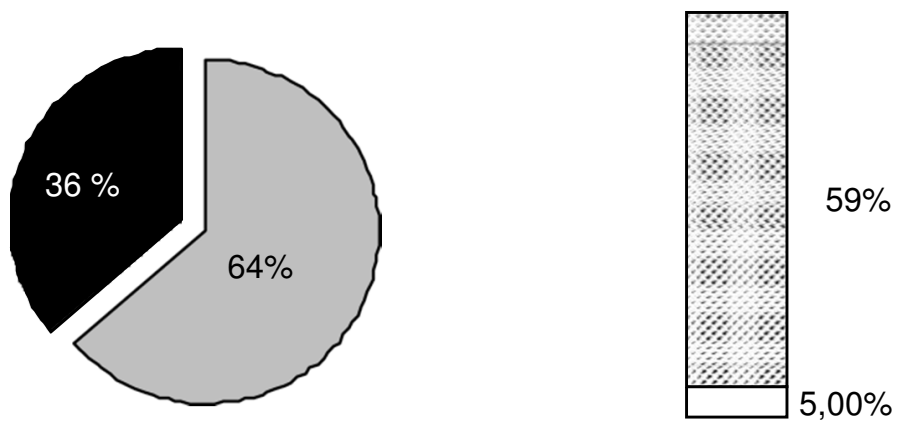

\section{Raíces cubiertas Raíces con daño en las aceras \\ Raíces expostas $\square$ Raíces sin daño en las aceras}

Figura 1 - Porcentaje de plantas que presentaban exposición de las raíces en la superficie del suelo, causando daños en las aceras. Barrio Jardín Maria de Queiroz, Campos dos Goytacazes, RJ, 2009.

El ancho de las aceras es uno de los factores determinantes para la selección de la especie de árbol y presenta una relación directa con su tamaño. Deben ser siempre observados los tipos de sistemas radiculares, siendo preferible los pivotantes profundos (MILANO y DALCIN, 2001). Posteriormente, se deben observar, con cuidado, las técnicas de plantío, la preparación del suelo y la fertilización, dando especial atención al collar o anillo ecológico, indispensable para el crecimiento del tronco y la infiltración del agua (SILVA et al., 2002; SCHUCH, 2006; PRADO y PAIVA, 2001).

Se observó que en un $84,11 \%$ de los casos, las plantas no presentaban en su entorno áreas de suelo libre para la captación de agua o crecimiento del tronco, llamado collar o anillo ecológico. En el 7,28\% de los casos los anillos fueron inferiores a $1 \mathrm{~m}^{2}$ y en el 8,61\% fueron mayores. Estos datos indican el desconocimiento de que se debe dejar como mínimo cerca de $0,30 \mathrm{~m}^{2}$ de área de suelo disponible para los árboles (LANDGRAF, PAIVA e PRADO, 2008) o $1 \mathrm{~m}^{2}$ o más quando se tiene uma amplia área de acera (CEMIG, 2001; ANDRADE, 2002; SILVA, 2003).

En el $67 \%$ de los casos, los árboles presentaban la altura de la primera bifurcación inferior a 1,80 m del suelo, solo la minoría $(10,60 \%)$ de los árboles no presentaban conflictos directos o significativos con el paso de peatones, por estar podados de un modo que facilitaba el camino. Se recomienda que las plantas sean llevadas a la siembra cuando se poden, después de su formación en el vivero y lleguen a una altura de 1,80 m, garantizando plantas estandarizadas que, además de no impedir el paso, presenten mejores condiciones para enfrentar las adversidades del medio urbano (CEMIG, 2001; LANDGRAF, PAIVA y PRADO, 2008).

Más de la mitad de los árboles observados (50,33\%), presentaban algún tipo de poda, de ellos el 15,23\% presentaban podas muy drásticas y fuera de las técnicas y fueron determinadas como mutilaciones (Figura 2). Las podas son necesarias solo cuando las copas ya se encuentran formadas, principalmente para su mantenimiento y limpieza, para reducir el sombreado, para evitar conflictos con el paso de peatones, con cables eléctricos aéreos y señalizaciones de tránsito (SCHUCH, 2006). 


\section{Podas y mutilaciones de los árboles}

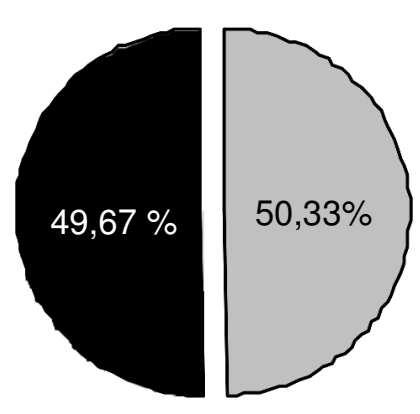

Árboles podados

Árboles sin podar

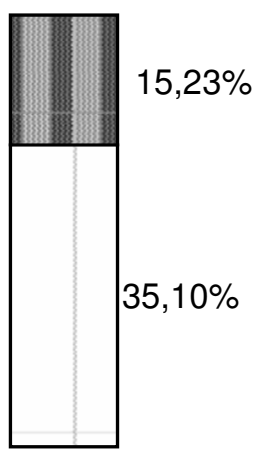

m Podas drásticas

Podas preventivas

Figura 2 - Porcentaje de plantas que presentaban podas y mutilaciones, Barrio Jardín Maria de Queiroz, Campos dos Goytacazes, RJ, 2009.

Las podas pueden ser divididas en las modalidades de: formación y poda programada preventiva. Las de formación, generalmente, se destinan a los viveros para que la planta alcance 1,80 m de altura en su primera bifurcación. Las podas programadas tienen, por objetivo, ajustar la planta al medio. Las leyes brasileñas determinan que las podas deben ser realizadas, solamente, con equipos autorizados por la alcaldía municipal o concesionarias de energía eléctrica. Las podas de emergencia están asociadas a riesgos de la energía o a peatones (LANDGRAF, PAIVA y PRADO, 2008).

Se observó que en un 42,39\% de los casos, las plantas estaban en conflicto con el medio, pois impedian el paso de los peatones en las aceras $(10,60 \%)$ o estaban en conflicto con la red de cables aéreos eléctricos o de comunicaciones (Figura 3). Es fundamental que previamente se haga un estudio sobre la disponibilidad del espacio para el tamaño y el tipo de copa de los árboles cuando adultos, para evitar drásticas interferencias en la arquitectura de las plantas y mutilaciones. 


\section{Incompatibilidad de la copa con el medio urbano}

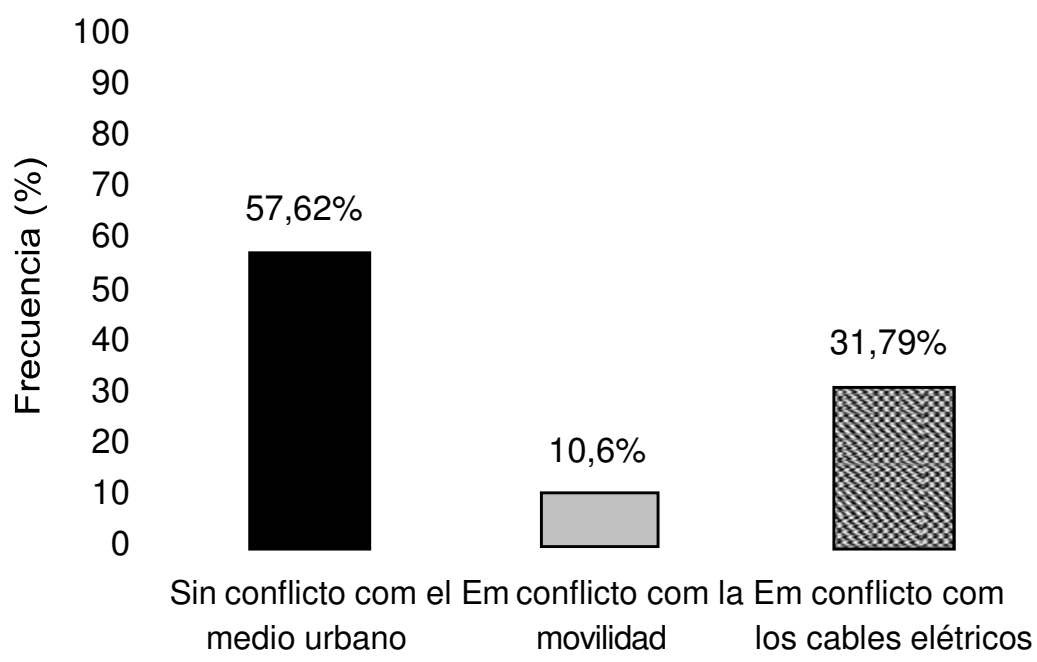

Figura 3 - Porcentaje de plantas que presentaban conflictos de la copa con el paso de peatones y redes de cables (eléctricos/telefónicos), Barrio Jardín Maria de Queiroz, Campos dos Goytacazes, RJ, 2009.

En áreas urbanas, los cortes y las podas son autorizados por los municipios, normalmente por las Secretarias de Agricultura y Medio Ambiente. Además de esto, los municipios deben tener una legislación específica, con el fin de auxiliarse. Las leyes que atribuyen a las alcaldías la responsabilidad sobre la realización de las podas de los árboles están en los Artículos 65 del Código Civil y el 151 del Código de Aguas.

Las leyes que determinan y regulan las áreas nativas de preservación y las especies de los árboles nativos inmunes al corte son: la Ley Federal № 4,771, de 15 de Septiembre de 1965 del Código Forestal y la Ley de Estatal No 8,518, de 21 de Enero de 1992 del Código Forestal del Estado.

La Constitución Federal de Brasil, prevé que todos los municipios con más de 20 mil habitantes deben, obligatoriamente, elaborar un Plan Maestro de forma democrática y participativa, bajo la aprobación de la Cámara Municipal. El Plan Maestro del Municipio de Campos dos Goytacazes (RJ, Brasil), en la Sección III de la Dimensión Ambiental, en el Artículo 15, plantea que las medidas y acciones prioritárias deben promover una reforma urbana y paisajística para la valoración del municipio, de acuerdo con su desarrollo regional.

La sustitución de especies que ya existen en la arborización de las calles de la ciudad, deben realizarse de una forma gradual y permanente. Ese proceso de sustitución debe ser precedido de amplia divulgación, por medio de diversos medio de comunicación, esclareciendo el plano de arborización y sus objetivos. Incluso parte de la arborización esta hecha sin los criterios de los moradores. Como se ve, existe la necesidad de respetar, con retrocesos mínimos, todas esas instalaciones para evitar transtornos futuros.

\section{CONCLUSIONES}

Este estudio observó, la relación de una especie nativa para 1,5 especies exóticas, el 57,72\% fueron principalmente, sibipiruna (Caesalpinia pluviosa DC.) y oiti (Licania tomentosa (Benth.) Fristch). Los árboles con raíces sin causar daños a las aceras totalizaron el 36\% de los casos y el 59\% presentaban problemas visibles de grietas, en un 5\% de los casos no se detectaron problemas. La disponibilidad de suelo libre al entorno de los árboles no fue respetada en un $84,11 \%$ de los casos. En el 8,61\% de los árboles, la zona ideal era igual o superior a $1,00 \mathrm{~m}^{2}$ y en el 7,28\% esa área era inferior. En el 33\% de los casos, los árboles presentaban una altura, en la primera bifurcación, igual o por encima de $1,80 \mathrm{~m}$ a partir del suelo, el 50,33\% de los árboles presentaban algún tipo de poda, siendo consideradas podas drásticas el 15,23\% de ellos. En el 10,60\% de los casos, los árboles impedian la movilidad y en el $31,79 \%$ existian conflictos con la red de cables eléctricos y de comunicaciones. 


\section{AGRADECIMIENTOS}

Los autores expresan sus sinceros agradecimientos a la señora Annabell Tamariz Hernandez por las aulas de Español que permitieron la redacción de este artículo.

\section{REFERÊNCIAS BIBLIOGRÁFICAS}

ANDRADE, T.O. Inventário e análise da arborização viária da estância turística de Campos dos Jordão, 2002. (Dissertação de Mestrado) - Escola Superior de Agricultura "Luiz de Queiroz", Piracicaba, São Paulo.

APG II. An update of the Angiosperm Phylogeny Group classification for the orders and families of flowering plants: APG II. Botanical Journal of the Linnean Society, 141:399-436, 2003.

BIONDI, D. e ALTHAUS. M. Árvores de rua de Curitiba: cultivo e manejo. Curitiba: FUPEF, 2005. 182 p.

CEMIG. Manual de arborização urbana. Gerais. Belo Horizonte. Cia de Energia de Minas Gerais. 2001, p.48.

DANTAS, I. C. e de SOUZA, C. M. C.. Arborização urbana na cidade de Campina Grande-PB: Inventário e suas especies. Revista de Biologia e Ciências da Terra, 4(2):1-6, 2004

DEL CARO, C.F.; OLIVEIRA, O.M.; SENNA, D.S.; LEMOS, R. e CALIMAN, J.P. Análise da arborização urbana das praças na região metropolitana da cidade de Alegre, ES. In: Encontro Latino Americano de Iniciação Científica, 8, e Encontro Latino Americano de Pós-Graduação, 9, 2009, São Paulo, Anais... Universidade do Vale do Paraíba, 2009: p.1-6.

GREY, G. W. e DENEKE, F. J. Urban forestry. New York: John Wiley, 1978, 279 p.

GUIZZO, D. J. e JASPER, A. Levantamento das especies arbóreas dos passeios das vias públicas do bairro Americano de Lajeado - RS, com indicação de problemas já existentes. Pesquisas Botânica, São Leopoldo, 56:185-208, 2005.

IBGE: Web Site do Instituto Brasileiro de Geografia e Estatística. Disponível em: <http://www.ibge.gov.br>. Acesso em 20 de dezembro de 2009. (Estatísticas de 2006).

KURIHARA, D. L.; IMAÑA-ENCINAS, J. e de PAULA, J. E. Levantamento da arborização do campus da Universidade de Brasília. Cerne, Universidade Federal de Larvas, 11(2):127-136, 2005.

LANDGRAF, P.R.C.; PRADO, N.J.S. e PAIVA, P.D.O. Arborização Urbana. Lavras: UFLA/FAEPE, 2008. 112p. Textos acadêmicos.

LANGOWSKI, e KLECHOWICH, N. Manual Prático de Poda e Arborização Urbana. Cianorte: APROMAC, 2001. 10p.

LINDENMAIER, D.S. e SANTOS, N.O. dos. Arborização urbana das praças de Cachoeira do Sul, RS, Brasil: fitogeografia, diversidade e índice de áreas verdes. Instituto Anchietano de Pesquisas de São Leopoldo, Pesquisa Botânica, 59: 307-320, 2008.

LORENZI, H. Árvores brasileiras: manual de identificação e cultivo de plantas arbóreas nativas do Brasil. v. 01. 5. ed. Nova Odessa - SP: Plantarum, 2008a.

LORENZI, H. Árvores brasileiras: manual de identificação e cultivo de plantas arbóreas nativas do Brasil. v. 02. 3. ed. Nova Odessa - SP: Plantarum, 2008 b. 
LORENZI, H. et al. Árvores exóticas no Brasil: madeireiras, ornamentais e aromáticas. Nova Odessa - SP: Instituto Plantarum, 2003.

MILANO, M.S. e DALCIN, E.C. Arborização de vias públicas. Rio de Janeiro: Ligth, 2001.

MILANO, M.S. e DISPERATI, A.A. Análise da quantidade e distribuição das áreas verdes no município de Curitiba - PR. Curitiba: UFPr, 1995. Apostila

MILANO, M.S. Arborização urbana. Curitiba: UFPr, 1995. Apostila.

MOURA, T.A. e SANTOS, V.L.L.V. Levantamento quali-quantitativo de especies arbóreas e arbustivas na arborização viária urbana dos bairros Centro e Centro Norte, Várzea Grande, Mato Grosso, Brasil, Soc. Bras. de Arborização Urbana (REVSBAU), Piracicaba, SP. 1(1): 97-117, 2009.

PAIVA, P.D.O. e GAVILAVES, M.L. Plantas ornamentais: classificação e uso em paisagismo. Lavras: UFLA/FAEPE,2004.97p. Textos acadêmicos.

PRADO, H.N. y PAIVA, P.D.O. Arborização urbana. Lavras: UFLA/FAETE, 2001.

SCHUCH, M.I.S.S. Arborização urbana: uma contribuição à qualidade de vida com uso de geotecnologias. 2006, (Dissertação de Mestrado). Universidade Federal de Santa Maria, Rio Grande do Sul.

SILVA JUNIOR, M.C. e CORREIA, C.R.M.A. Arborização no Campus da Universidade de Brasília: In: Encontro Nacional de Arborização Urbana, 2001, Brasília, D.F. Anais... Brasília DF, 2001.p.26.

SILVA, A. G. Inventário de arborização urbana viária: métodos de amostragem, tamanho e forma de parcelas. 2003, 110 p. (Dissertação de Doutorado)- Ciência Florestal, Universidade Federal de Viçosa, MG.

SILVA, E.M. et al.Estudo da arborização urbana do bairro Mansour na cidade de Uberlandia, MG. Caminhos da Geografia 3(5):73-83, 2002.

TEIXEIRA, I.F. Análise qualitativa da arborização de ruas do conjunto habitacional Trancredo Neves, Santa Maria-RS. Ciência Florestal, 9 (2): 9-21, 1999. 